\title{
Selection of Respiratory Mutants of Neurospora crassa
}

\author{
DAVID L. EDWARDS, FABIA KWIECINSKI, AND JONATHAN HORSTMANN
}

Department of Biological Sciences, State University of New York at Albany, Albany, New York 12222

Received for publication 12 January 1973

\begin{abstract}
A method is described that permits the isolation of mutants that are defective in mitochondrial respiration. The techniques of inositol-less death and overlay with 2,3,5-triphenyl-2H-tetrazolium chloride are utilized to select for mutant colonies. Colonies that survive inositol-less death and fail to reduce the tetrazolium dye are then tested polarographically for cyanide-sensitive respiration. A preliminary characterization of three mutants obtained by this method is presented. The mutants have been characterized by their cyanide-sensitive respiration rate, growth rate, the state III respiration rate of isolated mitochondria, inhibition of the respiration of isolated mitochondria by cyanide and antimycin A, and cytochrome spectra. All of the mutants described differ from the parent strain in some of these aspects.
\end{abstract}

The mitochondrial inner membrane of the fungus Neurospora crassa is a complex biological structure that receives genetic input from both the nuclear and mitochondrial genomes (22). The respiratory chain associated with this membrane has the characteristic $b-, c-$, and $a$-type cytochromes that are seen in mitochondria from higher organisms $(13,21)$. The haploid nature of Neurospora makes it an ideal organism for genetic studies, and the respiratory chain, with its easily observable cytochromes and characteristic enzyme activities, is an attractive membrane system for studies of the genetic control and assembly of membrane structures. Mutants of Neurospora that have abnormal respiratory systems have been isolated and extensively studied $(3,4,11,16,18$, 19, 22). Many of these mutations have been extrachromosomal in nature $(1,2,7,15,16)$. Progress has been slowed in this area, however, in that whereas those mutants that have been isolated have been extensively studied, a general method for isolating new mutants in which the respiratory chain is altered has not been available (2). We report on a method for selecting respiratory mutants of $N$. crassa that we developed in our laboratory and present a preliminary characterization of three mutants we isolated by using this method.

\section{MATERIALS AND METHODS}

Strains. An inositol-requiring strain, inos-89601, was obtained from the Fungal Genetics Stock Center,
Arcata, Calif. This strain was used in all mutation studies reported here and is referred to in the text as the parent strain.

Growth of cells. All cells were grown on a New Brunswick gyratory shaker at $30 \mathrm{C}$ in Vogel minimal medium (20) containing either $2 \%$ sucrose (sucrose minimal) or $50 \mathrm{mM}$ sodium acetate (acetate minimal) as a carbon source and supplemented with $50 \mu \mathrm{g}$ of inositol per $\mathrm{ml}$.

Selection of mutants. A protocol for the selection of respiratory mutants which we successfully used in our laboratory is shown below. It consists of several steps which are as follows.

(i) Inositol-less death. A modification of the method of Flavell and Fincham (5) was used. A 10-ml suspension of inos -89601 conidia in sterile water at a concentration of $10^{7}$ conidia per ml was irradiated with ultraviolet light to $50 \%$ killing. The suspension was then transferred into a 250 -ml flask to give a final volume of $40 \mathrm{ml}$ of acetate minimal medium plus inositol. The solution was shaken at $30 \mathrm{C}$ for $4 \mathrm{~h}$ and cells were reisolated on a Millipore filter, washed three times with sterile water, and transferred to a 1-liter flask containing $300 \mathrm{ml}$ of acetate minimal medium minus inositol. The flask was shaken at $30 \mathrm{C}$ for $48 \mathrm{~h}$. At the end of the incubation period, cells were reisolated on a Millipore filter, resuspended in sterile water, and plated on 50 to 100 petri plates of sorbose medium consisting of Vogel minimal medium, $1 \%$ sorbose, $0.05 \%$ glucose, $0.05 \%$ fructose, $2 \%$ agar, and $50 \mu \mathrm{g}$ of inositol per $\mathrm{ml}$. The plates were incubated at $30 \mathrm{C}$ for 2 to 3 days.

(ii) Tetrazolium overlay. Colonies which appeared on the plates were then overlayed with a solution containing $0.1 \mathrm{M}$ sodium phosphate $(\mathrm{pH}$ $7.0), 1.5 \%$ agar, and $0.5 \%$ 2,3,5-triphenyl-2H-tetrazolium chloride (Eastman). The agar-buffer solu- 
tion was cooled to $50 \mathrm{C}$ and the tetrazolium was added just before the overlay. The plates were then incubated at $39 \mathrm{C}$ for $3 \mathrm{~h}$. At the end of this time period, colonies were scanned visually and those that failed to reduce tetrazolium (i.e., remained colorless) were selected and placed on sucrose minimal plus inositol slants.

(iii) Replating. Conidia from the above individual isolates were replated on sorbose plates and were again overlayed with tetrazolium. The process was repeated until all of the colonies on the plate were colorless. If a mixture of colored and colorless colonies appeared, a colorless colony was selected and transferred to a slant, and the replating process was repeated. In our laboratory this process has been repeated 2 to 5 times. Isolates that remained mixed after five replatings have been discarded.

(iv) Respiration measurements. Liquid shaking cultures of the isolates obtained from the tetrazolium overlay procedure were grown at $30 \mathrm{C}$ in sucrose minimal medium plus inositol starting with an initial innoculum of $10^{5}$ conidia per $\mathrm{ml}$. Samples from the culture were removed at various time intervals, collected on a Millipore filtration apparatus using a Whatman no. 1 filter disc, and resuspended in $3 \mathrm{ml}$ of air-saturated sucrose minimal medium plus inositol. The oxygen consumption of the culture was then measured polarographically in a YSI model 53 biological oxygen monitor. The mycelial mass was then harvested quantitatively, dried at $60 \mathrm{C}$, and the dry weight was determined. Respiration rates are reported as microliters of oxygen consumed per hour per milligram of dry weight. Cyanide-sensitive respiration is calculated as the difference between the respiration rate in sucrose minimal medium plus inositol and the rate in the same medium plus millimolar potassium cyanide.

Isolation of mitochondria. Mitochondria for respiration measurements were isolated by grinding mycelium with $0.2-\mathrm{mm}$ diameter glass beads (3M Scotchlite) in a buffer consisting of $0.25 \mathrm{M}$ sucrose, $0.005 \mathrm{M}$ ethylenediaminetetraacetic acid, and $0.15 \%$ serum albumin, pH 7.0 (10). Portions (10 g) of mycelium were ground with an equal weight of glass beads for $90 \mathrm{~s}$ with a mortar and pestle at $4 \mathrm{C}$. A crude mitochondrial pellet was obtained by differential centrifugation between 2,000 and $20,000 \times g$, resuspended in isolation buffer, and further purified by centrifugation on a 20 to $65 \%$ sucrose gradient. Mitochondria from the sucrose gradient were used immediately for respiration measurements.

Mitochondria for spectral determinations were isolated by using the Glusulase method of Greenawalt et al. (9) and purified through two rounds of differential centrifugation.

Inhibitors. Antimycin A (Sigma) was dissolved in absolute ethanol and made fresh for each experiment. Potassium cyanide (Baker) was prepared fresh for each experiment in aqueous solution at $\mathrm{pH}$ 6.9.

Miscellaneous. Protein was determined by the method of Lowry et al. (14). Cytochrome spectra were obtained at room temperature on a Cary model 15 recording spectrophotometer. Mitochondria were disrupted with $1 \%$ Triton X-100 immediately before measurements were made. Difference spectra of dithionite-reduced minus oxidized samples were recorded. State III respiration rates were determined by utilizing the condition described by Hall and Greenawalt (10). Growth tube experiments were carried out as described by Bertrand and Pittenger (1).

\section{RESULTS}

A plot of the rate of cyanide-sensitive respiration as a function of time after germination for the parent strain, inos-89601, and three mutants derived from it by the procedure described in Materials and Methods is shown in Fig. 1. We have arbitrarily defined cyanidesensitive respiration as respiration which is inhibited by millimolar cyanide. This concentration of cyanide is sufficient to inhibit the respiration in wild-type cultures of Neurospora by greater than $90 \%$ (13) and the respiration of isolated wild-type mitochondria by $100 \%$. All of the mutants have rates of cyanide-sensitive respiration that are significantly lower than the parent strain from which they were derived. Mutant cni-1 exhibits no cyanidesensitive respiration after $24 \mathrm{~h}$ of growth. Oxygen electrode recordings showing the effect of millimolar cyanide on the respiration of 24-h cultures of these strains are shown in Fig. 2. Two of the mutants have been designated $c n i-1$ and cni-2 because they have high rates of cyanide-insensitive respiration. Mutant resp-1 does not have high cyanide-insensitive respiration but respires more slowly than the parent strain. Rates of growth in

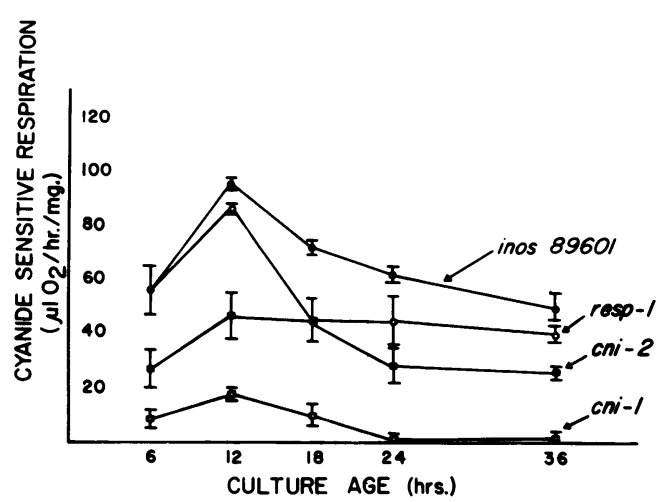

FIG. 1. Cyanide-sensitive respiration rates of the parent strain and three mutants derived from it. Cultures were inoculated at $10^{5}$ conidia/ml and grown at $30 \mathrm{C}$. At the various time points indicated, samples were taken from the cultures and the rates of cyanide-sensitive respiration were determined. The points indicate the mean of at least three determinations. The bars indicate the standard deviation of the mean. 


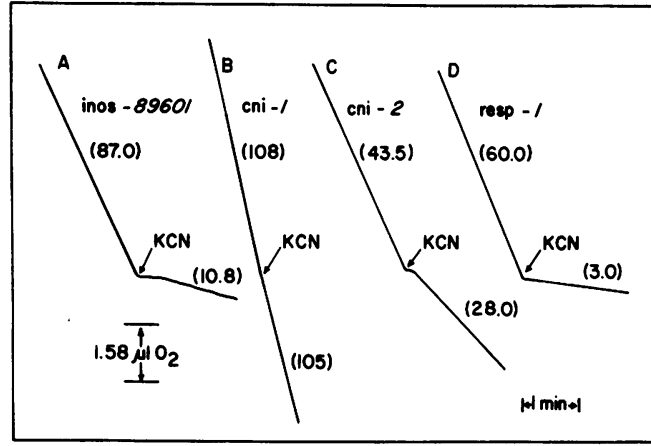

Fig. 2. Oxygen electrode recordings of 24- $h$ cultures of the parent strain and the three mutants. At the point indicated by the arrows, sufficient $K C N$ was added to give a final concentration of $1 \mathrm{mM}$. Values in parentheses are rates of oxygen uptake in microliters of $\mathrm{O}_{2}$ per hour per milligram of dry weight.

growth tubes for these four strains on both sucrose and acetate minimal medium plus inositol are shown in Fig. 3. It can be seen that all mutant strains grow more slowly than the parent strain on either sucrose or acetate medium. The mutant strains all grow poorly on acetate medium. Mutants cni-2 and cni-1 stopped growing altogether on acetate medium after 3 and 4 days of growth, respectively. The growth of the mutants was not appreciably enhanced on complete medium plus inositol.

To demonstrate that the results observed are due to alterations in mitochondrial respiration, we measured the rates of respiration of mitochondria isolated from these strains. The rates of oxidation of reduced nicotinamide adenine dinucleotide (NADH) and succinate measured in the presence of adenosine diphosphate (state III respiration) are shown in Table 1 . Values obtained for the mutants vary widely from those of the parent strain. None of the mutants oxidizes both of these substrates at the same rate as the parent strain. Oxidation rates that are both higher and lower than those observed for mitochondria from the parent strain are seen. The oxidation rates of the mutants are generally higher with NADH as a substrate and lower with succinate than those of the parent strain. Some of the mutants did not exhibit respiratory control when adenosine diphosphate was added to the respiring mitochondria. Such cases have been denoted by an asterisk ${ }^{*}$ ). It is not possible to distinguish between the absence of a phosphorylating system in these mutants and one that has been damaged during isolation. These cases, however, represent a marked difference between the phosphorylating system of the mutants and that of the parent strain. The lability of the phosphorylating system of poky mitochondria has been discussed by Eakin and Mitchell (3).

The levels of inhibition of respiration of isolated mitochondria by millimolar concentrations of cyanide or antimycin A are shown in Table 2. Whereas this concentration of inhibitors is sufficient to inhibit the respiration of wild-type and resp-1 mitochondria completely, it is not sufficient to inhibit the respiration of cni-1 and cni-2 mitochondria. The respiration of these mitochondria is inhibited by about 50 and $80 \%$, respectively. To determine whether the cyanide- and antimycin A-insensitive respiration of these two strains might be due to the impermeability of the mitochondrial membrane system to these inhibitors, the mitochondria were treated for $30 \mathrm{~s}$ in a Branson Sonifier (model W185D), by using a micro tip and a power output of five, and the effects of

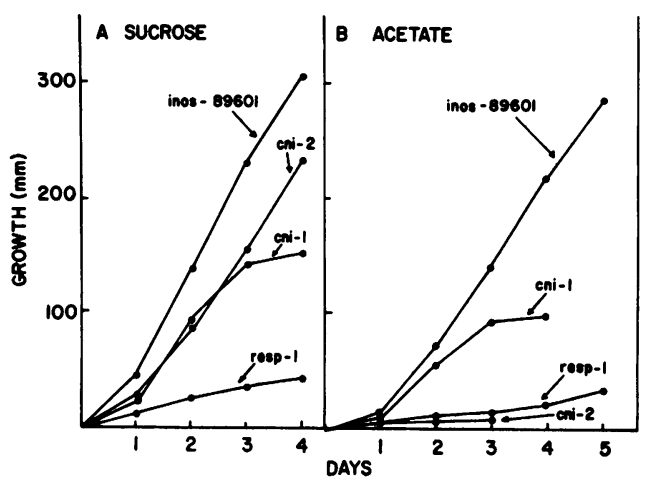

Fic. 3. Growth rates in growth tubes of the parent strain and the three mutants. The growth tubes were incubated at $30 \mathrm{C}$.

TABLE 1. State III respiration rates of isolated mitochondria ${ }^{a}$

\begin{tabular}{l|c|c}
\hline \multirow{2}{*}{ Mitochondria } & \multicolumn{2}{|c}{$\begin{array}{c}\text { Respiration rate } \\
\text { ( } \mu \text { atoms of O per min per mg) }\end{array}$} \\
\cline { 2 - 3 } & NADH & Succinate \\
\hline inos-89601 & $0.45 \pm 0.06$ & $0.37 \pm 0.03$ \\
cni-1 & $0.48 \pm 0.05$ & $0.19 \pm 0.06^{*}$ \\
cni-2 & $0.67 \pm 0.07$ & $0.23 \pm 0.04$ \\
resp-1 & $0.69 \pm 0.10^{*}$ & $0.17 \pm 0.04^{*}$ \\
\hline
\end{tabular}

${ }^{a}$ Values are reported as the mean \pm standard deviation of the mean for at least three determinations. Those values marked by an asterisk $\left({ }^{*}\right)$ indicate that the mitochondrial suspension did not exhibit respiratory control upon addition of adenosine diphosphate to the medium. Temperature was $30 \mathrm{C}$. 
TABLE 2. Inhibition of mitochondrial respiration by antimycin $A$ and cyanide ${ }^{a}$

\begin{tabular}{l|c|c|c|c}
\hline \multirow{2}{*}{ Mitochondria } & \multicolumn{4}{|c}{ Inhibition of respiration (\%) } \\
\cline { 2 - 5 } & \multicolumn{2}{|c|}{ Succinate plus: } & KCN & Antimycin A \\
\cline { 2 - 5 } & KCN & Antimycin A & NADH plus: \\
\hline inos-89601 & 100 & 100 & $93.9 \pm 3.9$ & $90.6 \pm 1.6$ \\
cni-1 & $51.2 \pm 2.0$ & $44.7 \pm 6.6$ & $40.0 \pm 9.6$ & $56.4 \pm 9.2$ \\
cni-2 & $80.0 \pm 2.4$ & $64.9 \pm 0.8$ & $83.4 \pm 3.6$ & $81.5 \pm 1.2$ \\
resp-1 & $98.0 \pm 2.0$ & $98.3 \pm 1.1$ & 100 & $99.2 \pm 0.2$ \\
\hline
\end{tabular}

${ }^{a}$ Respiration of isolated mitochondria was measured by using either succinate or NADH as a substrate (10). After a steady rate of respiration was attained, $\mathrm{KCN}$ or antimycin $\mathrm{A}$ was added to give a final concentration of $1 \mathrm{mM}$. The percent inhibition of the initial rate was determined. Values are reported as the mean \pm standard deviation of the mean for at least three determinations. Temperature was $30 \mathrm{C}$.

the inhibitors were redetermined. No significant deviation from the initial results was seen.

Cytochrome spectra of isolated mitochondria from the four strains are shown in Fig. 4. Mutants cni-2 and resp-1 show the presence of typical $b-, c-$, and $a$-type cytochromes and are qualitatively indistinguishable from mitochondria of the parent strain. Mutant cni-1, however, is deficient in cytochrome $a a_{3}$ and has an excess of cytochrome $c$. In this respect, the cytochrome content of this mutant is much like that of poky (13). It is difficult to determine from this spectrum whether or not cytochrome $b$ is present. A detailed analysis of mutant cni-1 will be the topic of a forthcoming communication (D. L. Edwards and F. Kwiecinski, manuscript in preparation).

\section{DISCUSSION}

The technique of inositol-less death on acetate medium has been utilized by Flavell and Fincham $(5,6)$ to select for acetate-nonutilizing (acu) mutants of Neurospora which were characterized as being defective in the enzymes of the Krebs cycle. To ascertain whether mutants of this type would survive our selection procedure, we obtained four of these mutants (acu-1, acu-5, acu-6, acu-7) from the Fungal Genetics Stock Center and overlayed colonies of them with tetrazolium as we have described. All of these acu mutants reduced tetrazolium and therefore would not have been selected by us as being respiratory mutants.

We found that approximately $30 \%$ of the mutants we selected as being unable to reduce tetrazolium have cyanide-sensitive respiration rates that are significantly different from the parent strain. The remaining $70 \%$ have cyanide-sensitive respiration rates that are indistinguishable from the parent strain. The inability of some of these colonies to reduce tetrazolium may be related to the Tet-R/Tet-W genes described by Gillie (8). Tet- $R$ is a gene which maps close to the mating-type locus and confers the ability to reduce tetrazolium. Tet-W is an allele of this gene that is unable to reduce tetrazolium. These genes have no effect on mitochondrial respiration and Tet-W is found in some wild-type strains. A mutation from Tet-R to Tet-W in our system would result in colonies unable to reduce tetrazolium but with normal mitochondrial respiration. Such colonies can only be eliminated in our system by screening for cyanide-sensitive respiration.

The high levels of cyanide- and antimycin A-insensitive respiration in cni-1 and cni-2 are

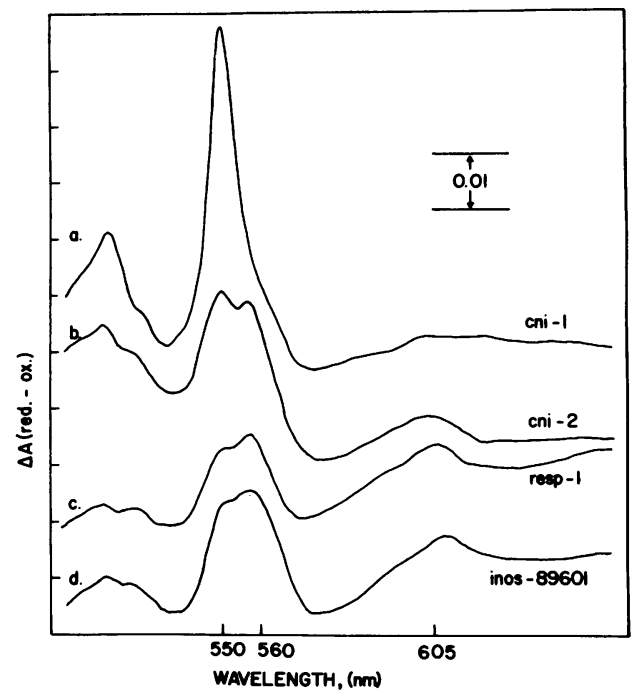

Fig. 4. Cytochrome difference spectra of isolated mitochondria from the parent strain and the three mutants. Reduction is with dithionite. Measurements were made at room temperature. Protein concentrations in milligrams per milliliter of the various fractions are: cni-1, 3.5; cni-2, 3.2; resp-1, 3.8; and inos-89601, 3.1 . 
of interest, as they may indicate the presence of branched or altered electron transport chains in these two mutants. A recent report by Lambowitz and Slayman (12) has shown that the poky strain of Neurospora has a high level of cyanide and antimycin A-insensitive respiration, and that this respiration can be inhibited by salicyl hydroxamic acid (SHAM), an inhibitor of cyanide-insensitive respiration in some higher plants (17). We confirmed these observations with poky in our laboratory and also tested mutants cni-1 and cni-2 with SHAM. We find that the cyanide-insensitive respiration of these mutants, either in whole mycelium or in isolated mitochondria, is also completely insensitive to a concentration of SHAM of $120 \mu \mathrm{g} / \mathrm{ml}$. This concentration of SHAM is sufficient to inhibit the cyanide-insensitive respiration of poky completely. These results suggest that at least two altered respiratory pathways can exist in Neurospora mitochondria, one which is sensitive to SHAM and one which is not.

The method described here appears to us to be a sound one for selecting mutants that have altered mitochondrial respiration. The difficulties in selecting for mitochondrial mutants in Neurospora have recently been discussed by Bertrand and Pittenger (2). The three mutants we described all have defective mitochondrial respiration yet all three differ in some of the respiratory properties of their mitochondria, suggesting that the lesions are all at different points in the respiratory chain with each having its own unique effect on the respiratory system. It may therefore be possible to obtain mutants at many points along the respiratory chain by using this method. An analysis of such mutants may be helpful in studies of the assembly of the respiratory chain. Studies along these lines are continuing in our laboratory.

\section{ACKNOWLEDGMENTS}

This work was supported by grants to D.L.E. from the National Science Foundation (GB-26319), the State University of New York Research Foundation, and Public Health Service grant GM-19410 from the National Institute of General Medical Sciences.

\section{LITERATURE CITED}

1. Bertrand, H., and T. H. Pittenger. 1969. Cytoplasmic mutants selected from continuously growing cultures of Neurospora crassa. Genetics 61:643-659.

2. Bertrand, H., and T. H. Pittenger. 1972. Isolation and classification of extranuclear mutants of Neurospora crassa. Genetics 71:521-533.

3. Eakin, R. T., and H. K. Mitchell. 1970. Alterations of the respiratory system of Neurospora crassa by the $m i-1$ mutation. J. Bacteriol. 104:74-78.

4. Edwards, D. L., and D. O. Woodward. 1969. An altered cytochrome oxidase in a cytoplasmic mutant of Neurospora. FEBS Lett. 4:193-196.

5. Flavell, R. B., and J. R. S. Fincham. 1968. Acetatenonutilizing mutants of Neurospora crassa. I. Mutant isolation, complementation studies, and linkage relationships. J. Bacteriol. 95:1056-1062.

6. Flavell, R. B., and J. R. S. Fincham. 1968. Acetatenonutilizing mutants of Neurospora crassa. II. Biochemical deficiencies and the roles of certain enzymes. J. Bacteriol. 95:1063-1068.

7. Garnjobst, L., J. F. Wilson, and E. L. Tatum. 1965. Studies on a cytoplasmic character in Neurospora crassa. J. Cell Biol. 26:413-425.

8. Gillie, O. J. 1970. Methods for the study of nuclear and cytoplasmic variation in respiratory activity of $\mathrm{Neu}$ rospora crassa and the discovery of three new genes. J. Gen. Microbiol. 61:379-395.

9. Greenawalt, J. W., D. O. Hall, and O. C. Wallis. 1967. Preparation and properties of Neurospora mitochondria, p. 142-146. In R. W. Estabrook and M. E. Pullman (ed.), Methods in enzymology, vol. 10. Academic Press Inc., New York.

10. Hall, D. O., and J. W. Greenawalt. 1964. Oxidative phosphorylation by isolated mitochondria of Neurospora crassa. Biochem. Biophys. Res. Commun. 17:565-569.

11. Haskins, F. A., A. Tissieres, H. K. Mitchell, and M. B. Mitchell. 1954. Cytochromes and the succinic acid oxidase system of poky strains of Neurospora. J. Biol. Chem. 200:819-826.

12. Lambowitz, A., and C. Slayman. 1971. Cyanide-resistant respiration in Neurospora crassa. J. Bacteriol. 108:1087-1096.

13. Lambowitz, A., C. Slayman, C. Slayman, and W. D. Bonner, Jr. 1972. The electron transport components of wild type and poky strains of Neurospora crassa. J. Biol. Chem. 247:1536-1545.

14. Lowry, O. H., N. J. Rosebrough, D. L. Farr, and R. J. Randall. 1951. Protein measurement with the Folin phenol reagent. J. Biol. Chem. 193:265-275.

15. McDougall, K. J., and T. H. Pittenger. 1966. A cytoplasmic variant of Neurospora crassa. Genetics 54:551-565.

16. Mitchell, M. B., and H. K. Mitchell. 1952. A case of "maternal" inheritance in Neurospora crassa. Proc. Nat. Acad. Sci. U.S.A. 38:442-449.

17. Schonbaum, G. R., W. D. Bonner, Jr., B. T. Storey, and J. T. Bahr. 1971. Specific inhibition of the cyanideinsensitive respiratory pathway in plant mitochondria by hydroxaminic acids. Plant Physiol. 47:124-128.

18. Tissieres, A., and H. K. Mitchell. 1954. Cytochromes and respiratory activities in some slow growing strains of Neurospora. J. Biol. Chem. 208:241-249.

19. Tissieres, A., H. K. Mitchell, and F. A. Haskins. 1953. Studies on the respiratory system of the poky strain of Neurospora crassa. J. Biol. Chem. 205:423-433.

20. Vogel, H. J. 1964. Distribution of lysine pathways among fungi: evolutionary implications. Amer. Natur. 98:435-446.

21: Weiss, H., G. von Jagow, M. Klingenberg, and T. Bucher. 1970. Characterization of Neurospora crassa mitochondria prepared with a grind-mill. Eur. J. Biochem. 14:75-82.

22. Woodward, D. O., D. L. Edwards, and R. B. Flavell. 1970. Nucleocytoplasmic interactions in the control of mitochondrial structure and function in Neurospora. Soc. Experimental Biol. 24:55-69. 